\title{
Correlations among Centrality Indices and a Class of Uniquely Ranked Graphs
}

DOI:

10.1016/j.socnet.2017.03.010

\section{Document Version}

Accepted author manuscript

Link to publication record in Manchester Research Explorer

\section{Citation for published version (APA):}

Schoch, D., Valente, T. W., \& Brandes, U. (2017). Correlations among Centrality Indices and a Class of Uniquely Ranked Graphs. Social Networks, 50, 46-54. https://doi.org/10.1016/j.socnet.2017.03.010

\section{Published in:}

Social Networks

\section{Citing this paper}

Please note that where the full-text provided on Manchester Research Explorer is the Author Accepted Manuscript or Proof version this may differ from the final Published version. If citing, it is advised that you check and use the publisher's definitive version.

\section{General rights}

Copyright and moral rights for the publications made accessible in the Research Explorer are retained by the authors and/or other copyright owners and it is a condition of accessing publications that users recognise and abide by the legal requirements associated with these rights.

\section{Takedown policy}

If you believe that this document breaches copyright please refer to the University of Manchester's Takedown Procedures [http://man.ac.uk/04Y6Bo] or contact uml.scholarlycommunications@manchester.ac.uk providing relevant details, so we can investigate your claim.

\section{OPEN ACCESS}




\title{
Correlations among Centrality Indices and a Class of Uniquely Ranked Graphs
}

\author{
David Schoch ${ }^{\mathrm{a}, *}$, Thomas W. Valente ${ }^{\mathrm{b}}$, Ulrik Brandes ${ }^{\mathrm{a}}$ \\ ${ }^{a}$ Department of Computer $\mathcal{G}$ Information Science, University of Konstanz, Germany \\ ${ }^{b}$ Department of Preventive Medicine, Keck School of Medicine, University of Southern \\ California, Los Angeles, CA, USA
}

\begin{abstract}
Various centrality indices have been proposed to capture different aspects of structural importance but relations among them are largely unexplained. The most common strategy appears to be the pairwise comparison of centrality indices via correlation. While correlation between centralities is often read as an inherent property of the indices, we argue that it is confounded by network structure in a systematic way. In fact, correlations may be even more indicative of network structure than relations among indices. This has substantial implications for the interpretation of centrality effects as it implies that competing explanations embodied in different indices cannot be separated from each other if the observed network has a star-like property.
\end{abstract}

Keywords: Network centrality, Correlation, Neighborhood inclusion, Centrality indices, Threshold graphs

\section{Introduction}

Plenty of centrality indices have been proposed to date and the list is everexpanding (Todeschini and Consonni, 2009). In addition to their application in empirical research, they are a frequent subject of methodological work aiming to provide a better understanding of what centrality indices measure and the theoretical foundations of the concept as a whole (Freeman, 1979: Sabidussi, 1966; Nieminen, 1974; Borgatti, 2005, Borgatti and Everett, 2006, Boldi and Vigna, 2014: Schoch and Brandes, 2016).

A frequently investigated question in this context deals with correlations among centrality indices (Bolland, 1988, Rothenberg, Potterat, Woodhouse,

We gratefully acknowledge financial support from the Deutsche Forschungsgemeinschaft under grant Br 2158/6-1 and the Graduate School of Decision Sciences at the University of Konstanz. A preliminary version of this research was presented at the Sunbelt XXXIV Social Networks Conference (St. Pete Beach, Florida, February 2014).

${ }^{*}$ Corresponding author

Email addresses: david.schoch@uni-konstanz.de (David Schoch), tvalente@usc.edu (Thomas W. Valente), ulrik.brandes@uni-konstanz.de (Ulrik Brandes) 
Darrow, Muth, and Klovdahl, 1995, Lee, 2006, Valente, Coronges, Lakon, and Costenbader, 2008, Batool and Niazi, 2014; Li, Li, Van Mieghem, Stanley, and Wang, 2015, Lozares, López-Roldán, Bolibar, and Muntanyola, 2015). The underlying assumption being that correlations are a consequence of the formal definition of indices and thus highlight differences in the conceptualization of centrality. High correlation "suggests considerable redundancy" (Bolland, 1988) and thus justifies, e.g., the use of a computationally less expensive index (Li) et al. 2015). Weakly correlated indices on the other hand "indicate distinctive measures likely to be associated with different outcomes" (Valente et al., 2008). It is thus not surprising that a correlation analysis is often performed when new indices are introduced to illustrate their disparity from existing measures (Newman, 2005, Estrada, Higham, and Hatano, 2009, Chen, Lü, Shang, Zhang, and Zhou, 2012, Benzi and Klymko, 2013).

Reported results, however, are often inconsistent with regard to the similarity of centrality indices. Bolland (1988) finds that closeness, degree and eigenvector centrality are substantially correlated, yet betweenness was comparatively uncorrelated. Rothenberg et al. (1995) use eight different indices on a network of HIV patients and determine that all measures are highly correlated, including degree and betweenness. Likewise, Lee (2006) observes a high correlation between degree and betweenness on a set of protein interaction networks. Based on a broader sample of networks of varying origin as well as a set of random graphs, Batool and Niazi (2014) find that, overall, closeness and eccentricity as well as degree and eigenvector centrality are highly correlated and that correlations with betweenness vary across networks.

These inconsistencies suggest that the role of the underlying network structure is far more important and profound than is accounted for. Indeed, structure appears to be of interest mostly when the stability of centrality indices in the face of missing data or sampled networks is investigated (Frantz, Cataldo, and Carley, 2009; Borgatti, Carley, and Krackhardt, 2006; Costenbader and Valente, 2003 Kim and Jeong, 2007). Borgatti et al. (2006) show that indices behave similar in terms of change patterns and level of robustness when edges are added or deleted on simple random graphs. Frantz et al. (2009) perform a similar analysis on more complex network structures and show that correlation varies with structure. Costenbader and Valente (2003) use a set of 60 empirical networks and examine the stability of indices on sampled networks and conclude that stability varies among indices. Again, they do concede that stability varies across networks.

Although many of the mentioned studies already point out that structural properties of networks such as density (Valente et al., 2008) or degree heterogeneity (Kim and Jeong, 2007) have an impact on the correlation among indices, the assumption that correlations mainly depend on formal definition prevails.

In contrast to previous work we show that correlations among indices may in fact be dominated by structural properties. These are not necessarily visible in common network statistics such as density or degree distribution, but related to the class of threshold graphs (Mahadev and Peled, 1995) which generalize the property of star graphs, ranking all vertices unambiguously. The relevance 
of this class is due to the preservation of its defining feature, completeness of the neighborhood-inclusion preorder, in all common centrality indices (Schoch and Brandes, 2016).

We start with a re-examination of a broad correlation study of Valente et al. (2008) (henceforth referred to as the Correlation Study), where varying correlations among a large set of indices were observed. Subsequently, we introduce the concept of neighborhood-inclusion and argue that the completeness of the ranking defined by neighborhood-inclusion is indicative for high correlations among indices, even for dual measures like betweenness and closeness (Brandes, Borgatti, and Freeman, 2016). We then illustrate this concept on the data of the Correlation Study and thus provide an alternative explanation for previously observed correlations. We conclude with implications of these findings for empirical research.

\section{Results of the Correlation Study}

Valente et al. (2008) use 60 networks compiled of eight different studies with varying context (Valente, 1995, Coleman, Katz, and Menzel, 1966; Burt, 1987, Rogers and Kincaid, 1981; Valente, Watkins, Jato, Van Der Straten, and Tsitsol, 1997). All studies were conducted in bounded communities by interviewing their members, asking about relationships with other members. A more detailed description of the data can be found in Valente et al. (2008). It is important to note that the studies greatly differ in size, number, type of questions asked and number of nominations allowed. This leads to a diverse set of networks with varying structural properties. Innately the networks are directed. The authors, however, also consider the undirected versions of the networks to compare symmetrized centrality measures. Our later analysis will focus on the undirected networks and the symmetrized indices, such that all results in this section for the directed cases are only given for the sake of completeness and replication purposes. Table 1 shows basic summary statistics of the eight studies and 60 directed networks.

\begin{tabular}{rrrrrrrr}
\hline study & networks & $\begin{array}{c}\text { average } \\
\text { size }\end{array}$ & $\begin{array}{c}\text { average } \\
\text { density }\end{array}$ & $\begin{array}{c}\text { average } \\
\text { outdegree }\end{array}$ & $\begin{array}{c}\text { symmetrized } \\
\text { centralization }\end{array}$ & $\begin{array}{c}\text { in-degree } \\
\text { centralization }\end{array}$ & $\begin{array}{c}\text { out-degree } \\
\text { centralization }\end{array}$ \\
\hline 1 & 3 & 64 & 0.06 & 2.61 & 0.15 & 0.19 & 0.12 \\
2 & 25 & 68 & 0.03 & 1.62 & 0.11 & 0.20 & 0.05 \\
3 & 11 & 76 & 0.03 & 1.94 & 0.16 & 0.29 & 0.06 \\
4 & 9 & 83 & 0.05 & 3.56 & 0.15 & 0.28 & 0.02 \\
5 & 9 & 82 & 0.50 & 39.17 & 0.30 & 0.15 & 0.49 \\
6 & 1 & 71 & 0.32 & 22.15 & 0.31 & 0.30 & 0.38 \\
7 & 1 & 72 & 0.20 & 14.19 & 0.18 & 0.24 & 0.28 \\
8 & 1 & 60 & 0.09 & 5.23 & 0.10 & 0.10 & 0.10 \\
\hline
\end{tabular}

Table 1: Characteristics of the networks compiled from various studies (replication of Table 2 from Valente et al. 2008).

Twelve different centrality indices are used in the correlation analysis. They include (in/out/symmetrized) degree, (in/out/symmetrized) closeness (Sabidussi 
1966), (in/out/symmetrized) betweenness (Freeman, 1977), eigenvector centrality (Bonacich, 1972) as well as integration and radiality (Valente and Foreman, 1998) and their joint symmetrized version. Correlations are assessed with Pearson's coefficient. Table 2 summarizes the average correlation among the twelve indices across the 60 networks.

\begin{tabular}{lrrrrrrrrrrrr}
\hline & 1 & 2 & $\mathbf{3}$ & 4 & $\mathbf{5}$ & 6 & 7 & $\mathbf{8}$ & 9 & 10 & $\mathbf{1 1}$ & $\mathbf{1 2}$ \\
\hline 1 indegree & & & & & & & & & & & & \\
2 outdegree & 0.28 & & & & & & & & & & & \\
3 degree & 0.81 & 0.73 & & & & & & & & & & \\
4 betweeness & 0.62 & 0.55 & 0.75 & & & & & & & & & \\
5 s-betweeness & 0.70 & 0.51 & $\mathbf{0 . 8 3}$ & 0.71 & & & & & & & & \\
6 closeness-in & 0.60 & 0.19 & 0.48 & 0.37 & 0.32 & & & & & & & \\
7 closeness-out & 0.20 & 0.82 & 0.58 & 0.40 & 0.37 & 0.07 & & & & & & \\
8 s-closeness & 0.45 & 0.63 & $\mathbf{0 . 6 2}$ & 0.37 & $\mathbf{0 . 4 0}$ & 0.50 & 0.64 & & & & & \\
9 integration & 0.74 & 0.28 & 0.61 & 0.50 & 0.42 & 0.93 & 0.18 & 0.57 & & & & \\
10 radiality & 0.23 & 0.87 & 0.62 & 0.45 & 0.40 & 0.12 & 0.98 & 0.66 & 0.23 & & & \\
11 s-int/rad & 0.50 & 0.69 & $\mathbf{0 . 6 9}$ & 0.43 & $\mathbf{0 . 4 7}$ & 0.52 & 0.68 & $\mathbf{0 . 9 9}$ & 0.60 & 0.71 & & \\
12 eigenvector & 0.74 & 0.70 & $\mathbf{0 . 9 1}$ & 0.67 & $\mathbf{0 . 6 9}$ & 0.46 & 0.54 & $\mathbf{0 . 5 7}$ & 0.60 & 0.59 & $\mathbf{0 . 6 5}$ & \\
\hline Average & 0.54 & 0.57 & 0.69 & 0.53 & 0.53 & 0.41 & 0.50 & 0.58 & 0.51 & 0.53 & 0.63 & 0.65 \\
Standard Deviation & 0.22 & 0.23 & 0.13 & 0.14 & 0.17 & 0.25 & 0.28 & 0.17 & 0.23 & 0.28 & 0.16 & 0.12 \\
\hline
\end{tabular}

Table 2: Average correlation between centrality indices across 60 networks. Symmetrized indices in bold. (replication of Table 3 from Valente et al. 2008).

Note that the symmetrized versions of degree, betweenness, closeness and eigenvector centrality are, on average, highly correlated. The larger differences between in and out measures can mostly be attributed to the nomination scheme of the individual studies, i.e. individuals nominate more people than they are themselves nominated.

Valente et al. (2008) conclude that the correlation between "degree, betweenness, closeness, and eigenvector indicates that these measures are distinct, yet conceptually related." While the results indeed point in this direction, individual differences on specific network structures are blurred out by averaging over all, or subsets of, networks. Also, as we argue below, Pearson's correlation coefficient is not an appropriate choice and might yield misleading results in this context. We thus re-evaluate the dataset in Section 5 with a rank based method and illustrate the impact of specific structural properties on correlations.

\section{Centrality and Uniquely Ranked Graphs}

Recently, it was shown that standard centrality indices can be expressed in a common framework based on path algebras (Schoch and Brandes, 2016). This generalization is used to show that common indices preserve what is called the neighborhood-inclusion preorder. In a simple undirected graph $G=(V, E)$, the neighborhood $N(i)$ of a node $i \in V$ is defined as the set of nodes $i$ is connected to, that is $N(i)=\{j:\{i, j\} \in E\}$. The closed neighborhood can then be defined as $N[i]=N(i) \cup\{i\}$. If the neighborhood $N(i)$ is contained in the 
closed neighborhood $N[j]$ (cf. Figure 1), then the centrality score of node $i$ will always be less or equal to the score of $j$.

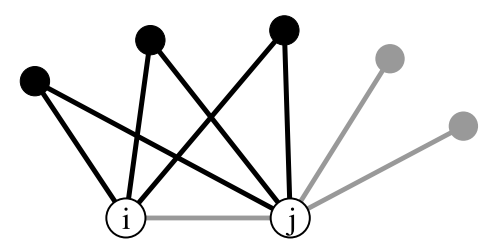

(a) $N(i) \subseteq N[j]$

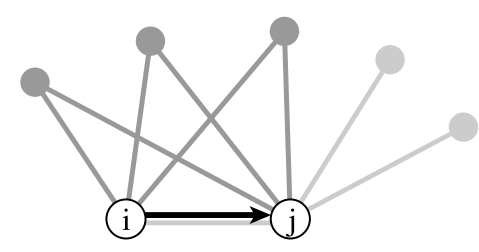

(b) $i$ dominated by $j$

Figure 1: (a) Example in which the neighborhood of $j$ includes that of $i$, independent of the presents of gray vertices and edges. (b) Neighborhood-inclusion relation represented as a directed graph; an edge $(i, j)$ indicates $N(i) \subseteq N[j]$ (underlying graph in gray for comparison).

More formally,

$$
N(i) \subseteq N[j] \Longrightarrow c(i) \leq c(j)
$$

holds for a great variety of indices $c$. Neighborhood-inclusion therefore induces a preorder (a reflexive and transitive binary relation) on the set of nodes which is respected by any centrality based node ranking. Figure 2 illustrates this preservation property on a simple example for degree, closeness, betweenness and eigenvector centrality.

(a)

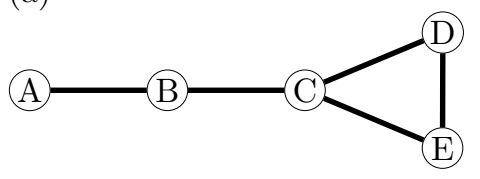

(c)

\begin{tabular}{c|cccc} 
scores & $\mathrm{dc}$ & $\mathrm{bc}$ & $\mathrm{cc}$ & $\mathrm{ec}$ \\
\hline $\mathrm{A}$ & 1 & 0 & 0.11 & 0.26 \\
$\mathrm{~B}$ & 2 & 3 & 0.17 & 0.57 \\
$\mathrm{C}$ & 3 & 4 & 0.20 & 1.00 \\
$\mathrm{D}$ & 2 & 0 & 0.14 & 0.82 \\
$\mathrm{E}$ & 2 & 0 & 0.14 & 0.82
\end{tabular}

(b)

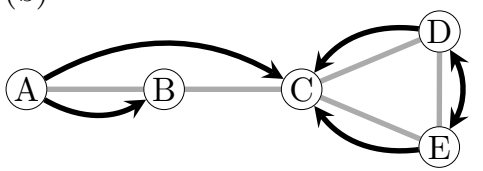

(d)

\begin{tabular}{c|cccc} 
ranks & $\mathrm{dc}$ & $\mathrm{bc}$ & $\mathrm{cc}$ & $\mathrm{ec}$ \\
\hline 1. & $\mathrm{C}$ & $\mathrm{C}$ & $\mathrm{C}$ & $\mathrm{C}$ \\
2. & $\mathrm{~B}, \mathrm{D}, \mathrm{E}$ & $\mathrm{B}$ & $\mathrm{B}$ & $\mathrm{D}, \mathrm{E}$ \\
3. & $\mathrm{~A}$ & $\mathrm{~A}, \mathrm{D}, \mathrm{E}$ & $\mathrm{D}, \mathrm{E}$ & $\mathrm{B}$ \\
4. & & & $\mathrm{~A}$ & $\mathrm{~A}$ \\
5. & & & &
\end{tabular}

Figure 2: (a) Example graph and (b) its neighborhood-inclusion preorder. (c) Centrality scores of degree (dc), betweenness (bc), closeness (cc) and eigenvector centrality (ec) of the graph and (d) their induced rankings.

This ordinal property amplifies the importance of centrality rankings compared to actual centrality scores. Effectively, scores have negligible meaning in empirical research, such that using rank-based correlation methods are more suitable for a correlation analysis. This is aggravated by the fact that Pearson's 
correlation coefficient is only fully applicable if two variables have a joined normal distribution (Embrechts, McNeil, and Straumann, 2002), which can not universally be assumed for centrality indices. Correlation analyses with Pearson's coefficient can thus lead to misleading results if the dependencies are monotone but nonlinear. For our later analysis, we use Kendall's $\tau$ as a rank based measure and Person's coefficient only to illustrate the differences.

A direct consequence of the preservation property is best understood by considering two extreme examples. Figure $3(\mathrm{a}, \mathrm{d})$ shows two graphs, (d) being totally ordered by neighborhood-inclusion, that is any pair of nodes can be ordered by neighborhood-inclusion, and (a) without any comparable pairs. In

(a)

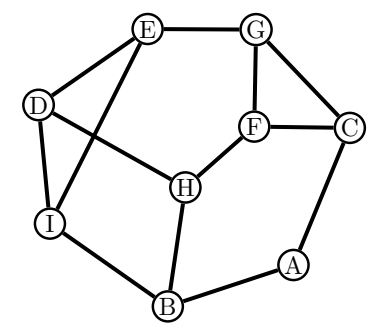

(d)

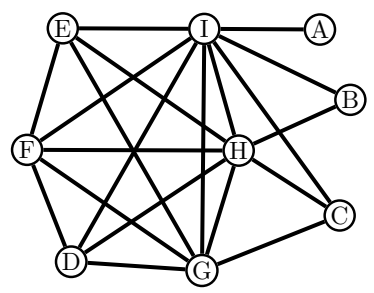

(b)

(D)

(I)

(e)

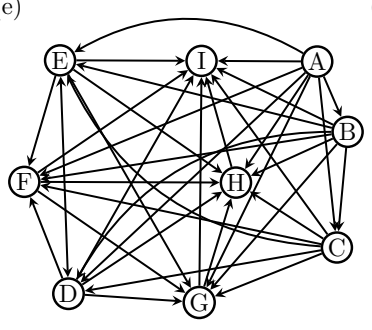

(c)

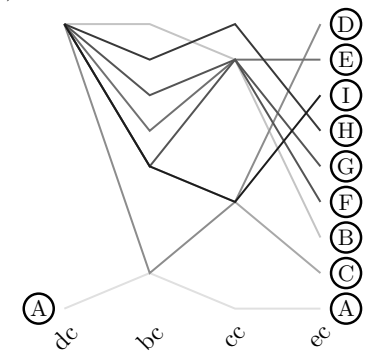

(f)

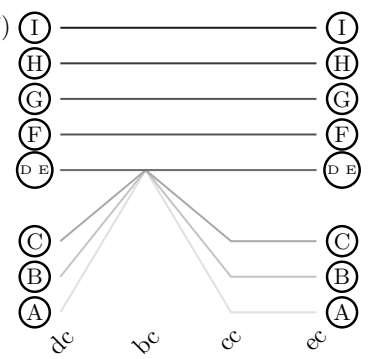

Figure 3: (a,d) Two example graphs with (b) empty and (e) total neighborhood-inclusion preorder. ( $\mathrm{c}+\mathrm{f})$ Parallel coordinates of induced rankings by centrality indices with ranking from top to bottom.

the former case, all centrality indices induce the same ranking (cf. Figure 3i ). Betweenness is a slight exception, since the preservation is not strict. That is, $N(i) \subseteq N[j]$ and $N(j) \nsubseteq N[i]$ only imply $c(i) \leq c(j)$ and not $c(i)<c(j)$. However, vertex $i$ will not be ranked higher than $j$. In the latter case, centrality rankings are completely undetermined, such that indices have the degree of freedom to rank vertices differently (cf. Figure 3r). In the following subsection, we give a characterization of a class of uniquely ranked graphs such as Figure 3(d).

\subsection{Threshold Graphs}

The class of graph for which the neighborhood-inclusion preorder is complete is known as threshold graphs (Mahadev and Peled, 1995). These graphs and their applications have been studied extensively in the literature in varying 
context (Chvátal and Hammer, 1977, Hammer, Ibaraki, and Simeone, 1981 Diaconis, Holmes, and Janson, 2008). The following theorem summarizes a number of alternative ways to characterize threshold graphs.

Theorem 1. Let $G=(V, E)$ be a simple undirected graph. Then, the following statements are equivalent.

(i) $G$ is a threshold graph.

(ii) There exist vertex weights $\omega: V \rightarrow \mathbb{R}_{0}^{+}$and a threshold $t \geq 0$ such that $\{u, v\} \in E \Longleftrightarrow \omega(u)+\omega(v)>t$.

(iii) $G$ can be constructed from the one-vertex graph by repeatedly adding an isolated vertex or a dominating vertex which is connected to every other vertex that has been added before.

(iv) $G$ does not contain the following graphs as induced subgraphs
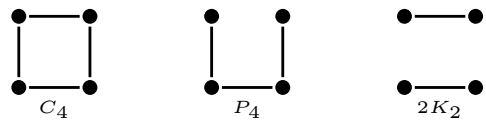

(v) $G$ is a split graph and the neighborhood of the independent set is nested.

The proof can be found in Mahadev and Peled (1995).

The degree sequence of a threshold graph is unigraphic, that is the structure of the graph is uniquely determined by its degree sequence up to node relabeling (Hammer et al., 1981). Theorem 1 $(v)$ implies that threshold graphs have a perfect core-periphery structure such that the node set can be partitioned into a clique and an independent set. Some basic examples for threshold graphs are shown in Figure 4.
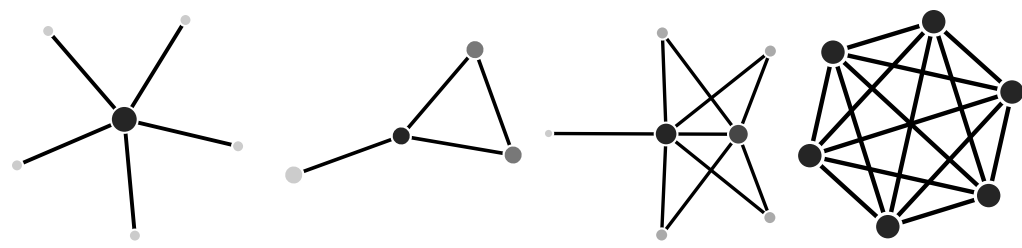

Figure 4: Examples of threshold graphs.

By definition, no two centrality indices that respect neighborhood-inclusion contradict each other on a threshold graph and the rankings are perfectly correlated, as illustrated in Figure 5.

We can, however, not expect to encounter threshold graphs in real-world social networks due to their rather artificial structure. Yet, we expect graphs close to being a threshold graph to have similar structural properties and thus exhibit high correlations among any pair of centrality indices. On the other 

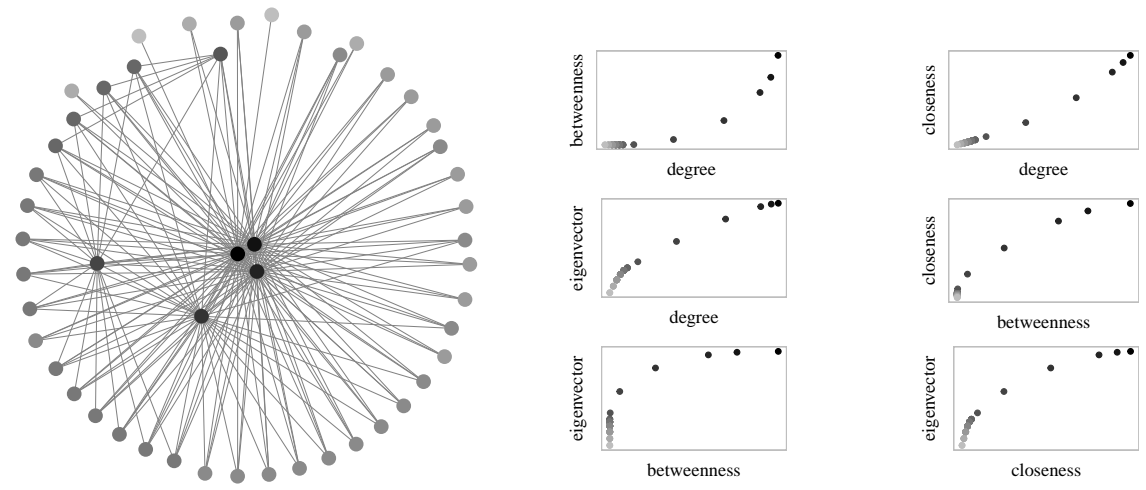

Figure 5: Threshold graph and pairwise centrality scores.

hand, the more distinct a graph is from a threshold graph the less determined the neighborhood-inclusion preorder will be, leading to more degrees of freedom and thus lower correlations. It is thus of importance to have reliable methods that allow the quantification of the distinctness between arbitrary graphs and threshold graphs.

\section{Distance to Uniquely Ranked Graphs}

In this section, we discuss several methods to assess how similar the structure of arbitrary graphs is to that of a threshold graphs.

Edit distance. The distance between two arbitrary graphs is commonly assessed with the graph edit distance, i.e. counting the minimum number of edge additions and deletions needed to turn one graph into the other (Gao, Xiao, Tao, and $\mathrm{Li}, 2010$ ). To determine how close an arbitrary graph is to being a threshold graph, we could thus determine the minimum number of edits necessary, to turn it into a threshold graph. This problem of threshold edit distance, however, was recently shown to be computationally intractable (Drange, Dregi, Lokshtanov, and Sullivan, 2015). Therefore, we have to rely on alternative measures or find bounds for the threshold edit distance.

Rewiring distance. A conceivable alternative to edit distance is to count the number of edge rewirings, that is, changing one endpoint of an edge to turn a graph into a threshold graph. This procedure will lead to a threshold graph with the same number of edges as the original graph. As Figure 6 illustrates, the closest threshold graph in terms of the threshold rewiring distance and the threshold edit distance can be distinct from each other.

The computational complexity of the threshold rewiring distance is unknown but it is most likely also an intractable problem to determine its minimum. Since an edge rewiring corresponds to two edits, we can at least state that the thresh- 


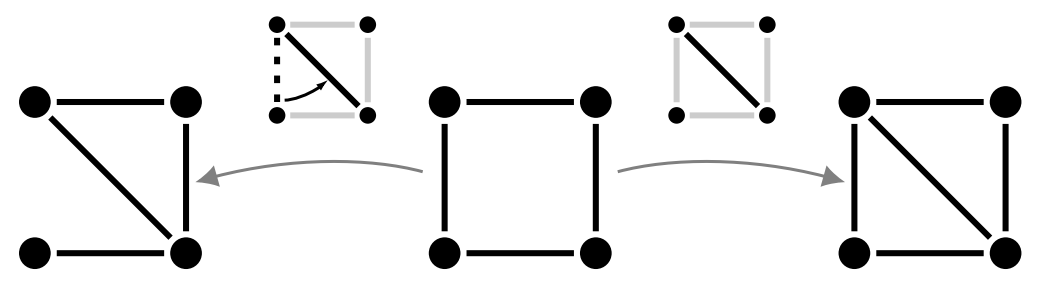

Figure 6: Illustration of the difference between edit and rewiring distance for a $C_{4}$ (middle). The left graph corresponds to the threshold graph with minimal rewiring distance and the right to the one with minimal edit distance. Both distances are equal to one on their respective scale.

old edit distance is bounded from above by twice the threshold rewiring distance.

Degree sequence editing. The threshold rewiring distance is closely related to two existing measures of non-thresholdness of degree sequences, the threshold gap gap by Hammer et al. (1981) and the majorization gap by Arikati and Peled (1994). Since both measures coincide (Mahadev and Peled, 1995), we here focus on the majorization gap. Given the degree sequence

$$
d=\left[d_{1}, d_{2}, \ldots, d_{n}\right] \text { with } d_{1} \geq d_{2} \geq \ldots \geq d_{n}
$$

of a graph with $n$ nodes, consider the corrected conjugated sequence $d^{\prime}$ defined by

$$
d_{k}^{\prime}=\left|\left\{i: i<k \wedge d_{i} \geq k-1\right\}\right|+\left|\left\{i: i>k \wedge d_{i} \geq k\right\}\right| \quad 1 \leq k \leq n .
$$

The majorization gap is then defined as

$$
\frac{1}{2} \sum_{k=1}^{n} \max \left\{d_{k}^{\prime}-d_{k}, 0\right\} \text {. }
$$

Informally, the majorization gap of $d$ is the minimum number of transformations, decreasing an entry by one and increasing another by the same amount, required to turn $d$ into a threshold sequence. This degree sequence edits are referred to as reverse unit transformations (Mahadev and Peled, 1995). Since multiple graphs can have the same degree sequence, a reverse unit transformation sometimes but not always correspond to rewiring an edge from one neighbor to another. One such case is shown in Figure 7. The majorization gap thus provides a lower bound for the threshold rewiring distance.

With the majorization gap, we therefore have a rough estimate for the edit distance of arbitrary graphs to their closest threshold graph. In the absence of a better alternative measure, we hereafter employ the majorization gap to quantify the structural dissimilarity of a graph from a threshold graph keeping the above points in mind. To make results comparable across networks, we normalize the majorization gap by the total number of edges in the networks. Hence, the normalized majorization gap is bounded between 0 (with equality for 


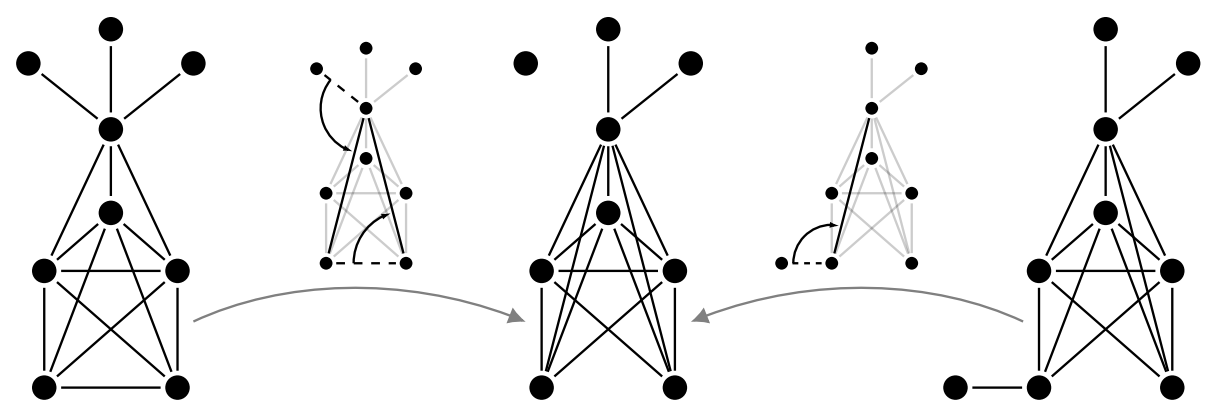

Figure 7: Illustration of the ambiguity of the majorization gap. The graphs on the left and right both have the same degree sequence $[6,5,5,5,4,4,1,1,1]$ and a majorization gap of 1. However, two edge rewirings are necessary for the left graph to obtain the threshold graph corresponding to the majorization gap (middle) and only one for the right graph.

threshold graphs) and 1, if all entries of the degree sequence have to be changed.

Number of comparable pairs. Besides the majorization gap, we assess the completeness of the neighborhood-inclusion preorder for each network. This measure serves as an indicator for the expectable association among indices. The more pairs of nodes are comparable, the higher are the correlations expected to be. On the other hand, if only a small fraction of nodes is comparable, then the correlations should display a higher variability.

Eigengap heuristic. As noted before, threshold graphs have an idealized coreperiphery structure. This suggests that graphs close to being a threshold graph exhibit characteristics inherent in core-periphery networks. There exist various approaches to detect and quantify the core-periphery structure of networks, e.g. by correlation with an idealized structure (Borgatti and Everett, 1999). We here employ an approach motivated by results from spectral graph theory.

For many existing graph clustering algorithms, an input parameter $k$ is required that specifies the number of clusters to be found. A way to determine this number a priori is the so called eigengap heuristic (von Luxburg, 2007). The adjacency matrix $A$ of a simple undirected graph can be decomposed into a set of orthogonal eigenvectors $x_{1}, x_{2}, \ldots, x_{n}$ each associated with an eigenvalue $\lambda_{1} \geq \lambda_{2} \geq \ldots \geq \lambda_{n}$. The number of presumable densely connected subgraphs can be estimated by investigating gaps in the eigenvalue sequence. The parameter $k$ is chosen such that $\left|\lambda_{i}-\lambda_{i+1}\right|$ is small for $1 \leq i<k$ and $\left|\lambda_{k}-\lambda_{k+1}\right|$ is comparably large. For core-periphery graphs, we can expect that a gap appears at $k=1$ since only one densely connected subgraph, the core, is present. A simplistic illustration of this assertion is shown in Figure 8. We use

$$
1-\frac{\lambda_{2}}{\lambda_{1}}
$$

as eigengap measure to keep results comparable across networks and such that higher values correspond to larger gaps. The maximum of this measure for 


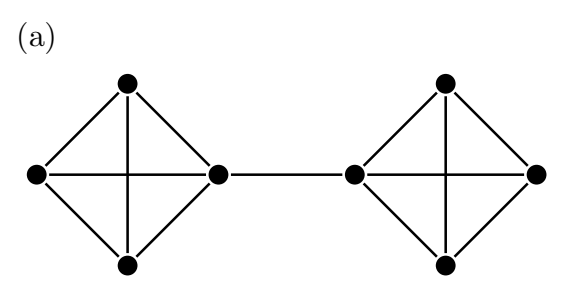

(c)

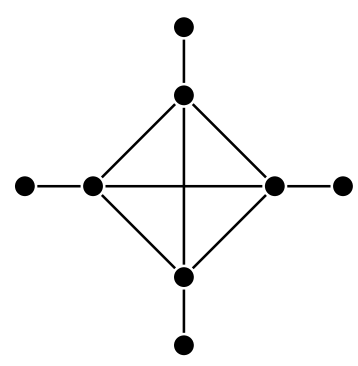

(b) eigenvalues

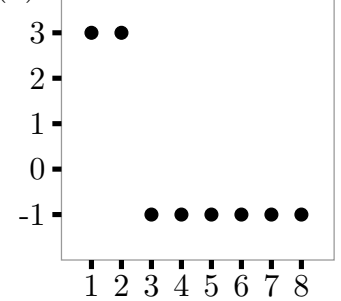

(d) eigenvalues

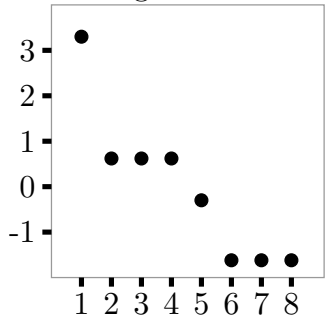

Figure 8: (a) graph with two cliques connected by one edge and (c) graph with clique as core and 4 peripheral nodes. (b) $+(\mathrm{d})$ the distributions of the 8 eigenvalues of the respective adjacency matrix with a gap at 2 for (a) and 1 for (c).

graphs with $n$ nodes is attained for the star graph where $\lambda_{1}=\sqrt{n-1}$ and $\lambda_{2}=0$.

Graph diameter. For any perfect core-periphery graph, the diameter is at most 3 (a path between two peripheral nodes via two core nodes) and exactly 2 if the graph is a threshold graph. Thus, the higher the diameter of a graph, the larger we expect the discrepancy to a threshold graph to be.

Figure 9 illustrates how the eigengap and the diameter covary with the majorization gap. We can see that the eigengap as well as the diameter are strongly correlated with the majorization gap. Although we argued that the majorization gap is only a distance estimate, it works reasonably well in assessing the "core-peripheriness" of a network, especially since it is computationally cheap also for very large networks.

Density. The sometimes conjectured association between density and centrality correlation is mitigated by the fact that threshold graphs exist at every density level from trees (e.g. a star graph) to cliques. Hence, we exclude the density from our analysis. 

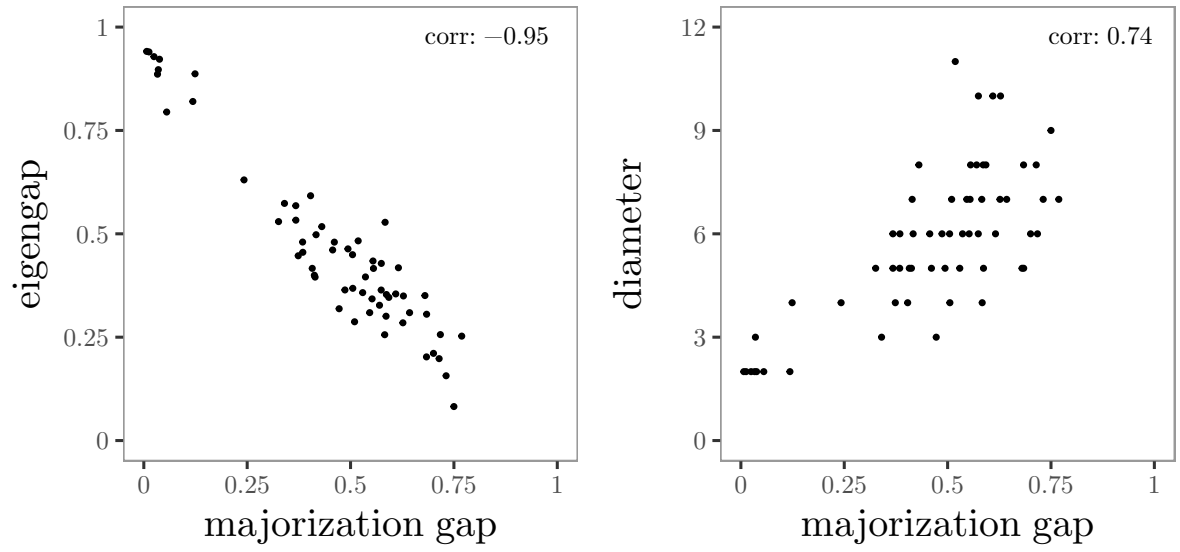

Figure 9: Network statistics that covary with the majorization gap.

\section{Results}

In contrast to the Correlation Study, we restrict ourselves to the undirected versions of the networks and delete all instances of isolated vertices. This is justified by the fact that we are only concerned with theoretical implications and do not use centrality as explanatory variables for any observed phenomena (i.e. centrality effects). Also, the concept of uniquely ranked graphs and the neighborhood-inclusion preorder presented in Section 3 were, so far, only explored for undirected graphs. Furthermore, we only compare the four prototypical indices degree (dc), closeness (cc), betweenness (bc) and eigenvector centrality (ec) to illustrate our assertions.

We argued in the last section, that Pearson's correlation coefficient is not necessarily the appropriate choice for measuring association among centrality indices. That is, observed varying correlations might simply be an artifact of the chosen coefficient. Figure 10, however, illustrates that this is not the case. The figure shows a comparison of Kendall's $\tau$ and Pearson's coefficient, displaying considerable differences between the two correlation measures in average correlation. Overall, Kendall's $\tau$ yields lower values, however, what remains is the wide range of values for each pair of indices. If we assume correlation to be primarily driven by formal connections, we would expect a much lower variability.

As a first structural test, we assess the completeness of the neighborhoodinclusion preorder for each network. Figure 11 illustrates how the fraction of comparable pairs affects the correlation. 


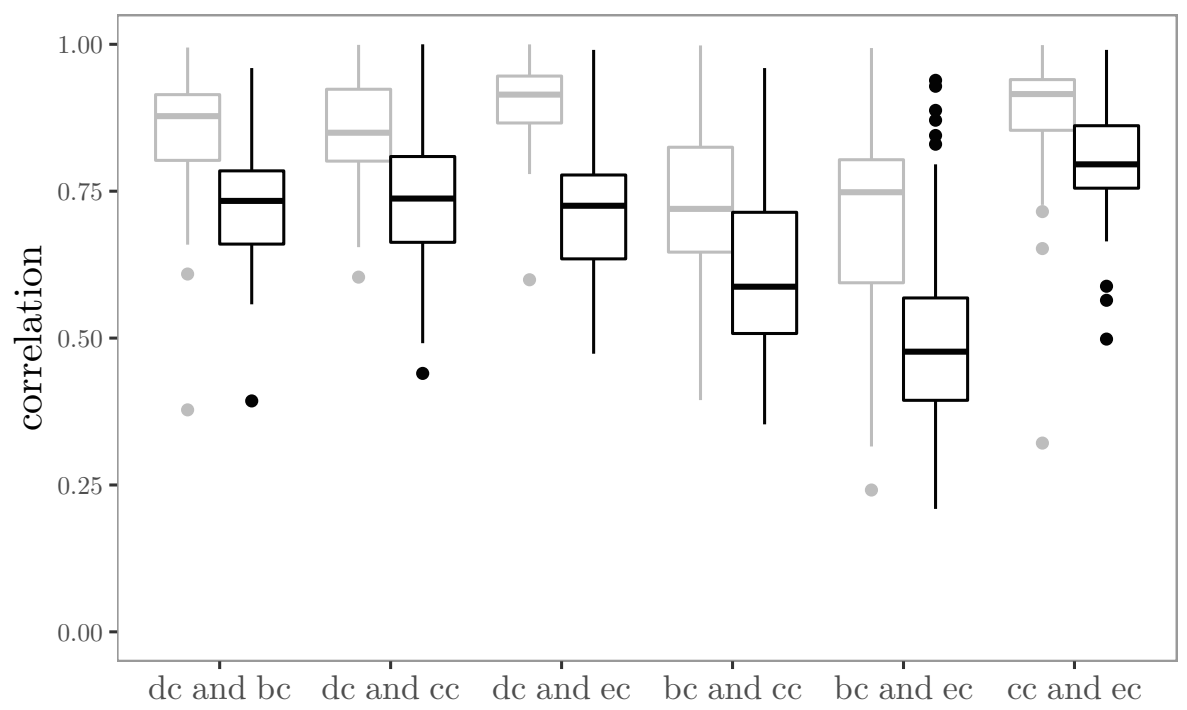

Figure 10: Boxplot of pairwise correlations on the 60 undirected networks. Kendall's $\tau$ in black and Pearson's coefficient in gray.

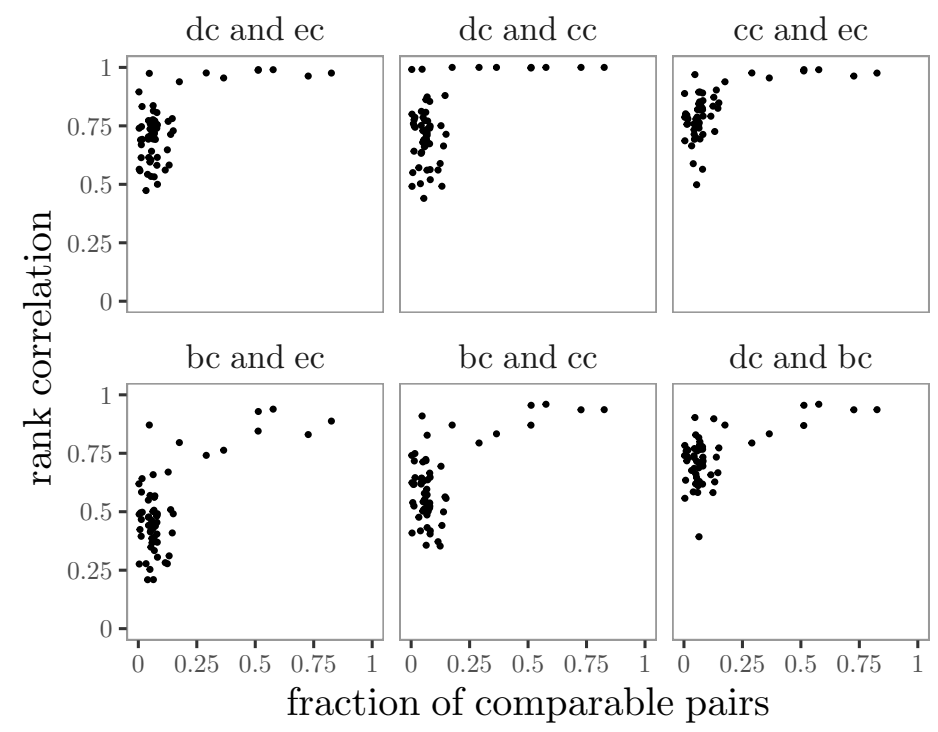

Figure 11: Rank Correlation and fraction of comparable pairs by neighborhood-inclusion for the 60 undirected networks.

Most of the graphs only have a very small fraction of comparable pairs, such that we can not draw any general conclusions about a connection between the 
completeness of the neighborhood-inclusion preorder and observed correlations. At least in the lower range it is apparent that the correlation can vary significantly due to the degree of freedom offered by a sparse neighborhood-inclusion preorder. Further, the few graphs with a more complete neighborhood-inclusion preorder display the anticipated outcome of generally higher correlations. More variety among the graphs can be observed when turning to the normalized majorization gap depicted in Figure 12.

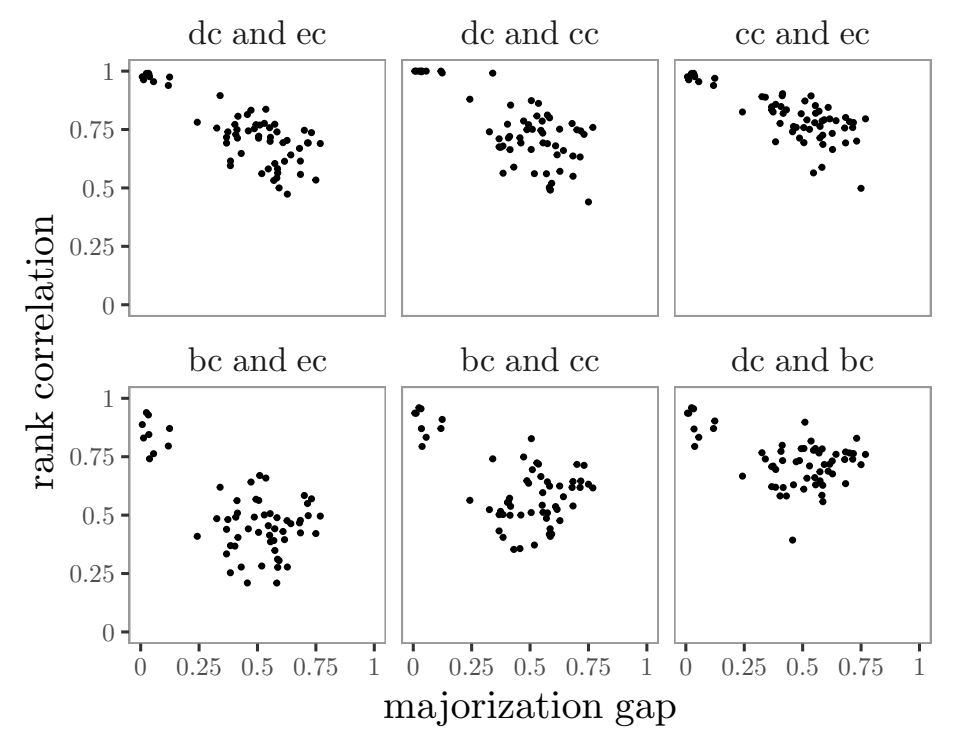

Figure 12: Rank Correlation and normalized majorization gap for the 60 undirected networks.

We can see that the correlations decline, more or less, with increasing majorization gap. This indicates that the distance to threshold graphs is indeed partly indicative for correlations among centrality indices. Individual differences, however, can still be observed. Especially the correlation of betweenness with other measures exhibits a higher variance, suggesting that further structural properties affect the correlations. Nonetheless, we observe that even conceptually different indices like betweenness and closeness can be highly correlated if the network is close enough to a threshold graph. In contrast, even indices that are suspected to be similar in a formal way, like degree and eigenvector centrality, can be quite uncorrelated if the network structure permits it.

\section{Conclusion}

We have shown that correlations among centrality indices are not necessarily indicative of their formal and conceptual similarity. The reason is that centrality indices induce the same ranking on threshold graphs. This class of 
graphs generalizes the star graph property and represent the purest form of a core-periphery structure. The closer any graph to a threshold graph, the more correlated centrality indices are, independent of their conceptual distinctness. To assess closeness to a threshold graph we used the majorization gap as a simple and computationally tractable alternative to edit distance.

Our findings have important implications for empirical research. If the structure of a network is close to that of a threshold graph, which will be the case for many core-periphery networks, a centrality effect cannot be explained with reference to the conceptual foundation of a specific centrality index because most other indices, with their competing explanations, will yield similar results.This hints at a trade-off between a pronounced centrality effect and the specificity of its explanation.

It is therefore important to study in more detail such notions of distance from threshold graphs that scale with the degree to which different centrality concepts diverge. To do so, however, we first need to understand which structural features cause the scores of different centrality indices to deviate. Do these features have natural generalizations in directed and valued networks? A problem closely related to the distance from uniquely ranked graphs is the sensitivity of indices with respect to structural changes. Since these may be caused by missing and erroneous data, it can be seen as a proxy for reliability.

As a consequence of our findings, future work on the comparison of existing, and development of new centrality indices should focus on the nature and prevalence of structural features that lead to distinct evaluations. Ideally, this would provide a handle on the confidence that can be placed in particular explanations of centrality effects.

\section{Bibliography}

Arikati, S. R., Peled, U. N., 1994. Degree sequences and majorization. Linear Algebra and its Applications 199, 179-211.

Batool, K., Niazi, M. A., 2014. Towards a methodology for validation of centrality measures in complex networks. PLoS ONE 9 (4), e90283.

Benzi, M., Klymko, C., 2013. Total communicability as a centrality measure. Journal of Complex Networks 1 (2), 124-149.

Boldi, P., Vigna, S., 2014. Axioms for centrality. Internet Mathematics 10 (3-4), $222-262$.

Bolland, J. M., 1988. Sorting out centrality: An analysis of the performance of four centrality models in real and simulated networks. Social Networks 10 (3), 233-253.

Bonacich, P., 1972. Factoring and weighting approaches to status scores and clique identification. Journal of Mathematical Sociology 2, 113-120. 
Borgatti, S. P., 2005. Centrality and network flow. Social Networks 27 (1), 5571.

Borgatti, S. P., Carley, K. M., Krackhardt, D., 2006. On the robustness of centrality measures under conditions of imperfect data. Social Networks 28 (2), $124-136$.

Borgatti, S. P., Everett, M. G., 1999. Models of core/periphery structures. Social Networks 21 (4), 375-395.

Borgatti, S. P., Everett, M. G., 2006. A graph-theoretic perspective on centrality. Social Networks 28 (4), 466-484.

Brandes, U., Borgatti, S. P., Freeman, L. C., 2016. Maintaining the duality of closeness and betweenness centrality. Social Networks 44, 153-159.

Burt, R. S., 1987. Social contagion and innovation: Cohesion versus structural equivalence. American Journal of Sociology 92, 1287-1335.

Chen, D., Lü, L., Shang, M.-S., Zhang, Y.-C., Zhou, T., 2012. Identifying influential nodes in complex networks. Physica A: Statistical Mechanics and its Applications 391 (4), 1777-1787.

Chvátal, V., Hammer, P. L., 1977. Aggregation of inequalities in integer programming. Annals of Discrete Mathematics 1, 145-162.

Coleman, J., Katz, E., Menzel, H., 1966. Medical Innovation: A Diffusion Study. The Bobbs-Merrill Company.

Costenbader, E., Valente, T. W., 2003. The stability of centrality measures when networks are sampled. Social Networks 25 (4), 283-307.

Diaconis, P., Holmes, S., Janson, S., 2008. Threshold graph limits and random threshold graphs. Internet Mathematics 5 (3), 267-320.

Drange, P. G., Dregi, M. S., Lokshtanov, D., Sullivan, B. D., 2015. On the threshold of intractability. In: Proceedings of the 23rd Annual European Symposium on Algorithms (ESA 2015). Vol. 9294 of Lecture Notes in Computer Science. Springer-Verlag, pp. 411-423.

Embrechts, P., McNeil, A., Straumann, D., 2002. Correlation and dependence in risk management: properties and pitfalls. In: Dempster, M. A. H. (Ed.), Risk Management: Value at Risk and Beyond. Cambridge University Press, pp. $176-223$.

Estrada, E., Higham, D. J., Hatano, N., 2009. Communicability betweenness in complex networks. Physica A: Statistical Mechanics and its Applications 388 (5), 764-774. 
Frantz, T. L., Cataldo, M., Carley, K. M., 2009. Robustness of centrality measures under uncertainty: Examining the role of network topology. Computational and Mathematical Organization Theory 15 (4), 303-328.

Freeman, L. C., 1977. A set of measures of centrality based on betweenness. Sociometry 40 (1), 35-41.

Freeman, L. C., 1979. Centrality in social networks: Conceptual clarification. Social Networks 1 (3), 215-239.

Gao, X., Xiao, B., Tao, D., Li, X., 2010. A survey of graph edit distance. Pattern Analysis and Applications 13 (1), 113-129.

Hammer, P. L., Ibaraki, T., Simeone, B., 1981. Threshold sequences. SIAM Journal on Algebraic Discrete Methods 2 (1), 39-49.

Kim, P.-J., Jeong, H., 2007. Reliability of rank order in sampled networks. The European Physical Journal B 55 (1), 109-114.

Lee, C.-Y., 2006. Correlations among centrality measures in complex networks. arXiv preprint physics, 0605220 .

Li, C., Li, Q., Van Mieghem, P., Stanley, H. E., Wang, H., 2015. Correlation between centrality metrics and their application to the opinion model. The European Physical Journal B 88 (3), 1-13.

Lozares, C., López-Roldán, P., Bolibar, M., Muntanyola, D., 2015. The structure of global centrality measures. International Journal of Social Research Methodology 18 (2).

Mahadev, N. V., Peled, U. N., 1995. Threshold Graphs and Related Topics. Vol. 56 of Annals of Discrete Mathematics. Elsevier.

Newman, M. E. J., 2005. A measure of betweenness centrality based on random walks. Social Networks 27 (1), 39-54.

Nieminen, J., 1974. On the centrality in a graph. Scandinavian Journal of Psychology 15, 332-336.

Rogers, E. M., Kincaid, D. L., 1981. Communication Networks: Toward a New Paradigm for Research. The Free Press.

Rothenberg, R. B., Potterat, J. J., Woodhouse, D. E., Darrow, W. W., Muth, S. Q., Klovdahl, A. S., 1995. Choosing a centrality measure: epidemiologic correlates in the colorado springs study of social networks. Social Networks 17 (3), 273-297.

Sabidussi, G., 1966. The centrality index of a graph. Psychometrika 31 (4), 581-603. 
Schoch, D., Brandes, U., 2016. Re-conceptualizing centrality in social networks. European Journal of Applied Mathematics 27 (6), 971-985.

Todeschini, R., Consonni, V., 2009. Molecular Descriptors for Chemoinformatics, 2nd Edition. Wiley-VCH.

Valente, T. W., 1995. Network models of the diffusion of innovations. Cresskill: Hampton Press.

Valente, T. W., Coronges, K., Lakon, C., Costenbader, E., 2008. How correlated are network centrality measures? Connections 28 (1), 16.

Valente, T. W., Foreman, R. K., 1998. Integration and radiality: Measuring the extent of an individual's connectedness and reachability in a network. Social Networks 20 (1), 89-105.

Valente, T. W., Watkins, S. C., Jato, M. N., Van Der Straten, A., Tsitsol, L.-P. M., 1997. Social network associations with contraceptive use among cameroonian women in voluntary associations. Social science \& medicine $45(5), 677-687$.

von Luxburg, U., 2007. A tutorial on spectral clustering. Statistics and Computing 17 (4), 395-416. 